Research Paper

\title{
miR-758-3p: a blood-based biomarker that's influence on the expression of CERP / ABCA1 may contribute to the progression of obesity to metabolic syndrome
}

\author{
Sadhbh O'Neill ${ }^{1}$, Mette Bohl Larsen², Søren Gregersen ${ }^{2}$, Kjeld Hermansen ${ }^{2}$ and \\ Lorraine $\mathbf{O}^{\prime}$ Driscoll ${ }^{1}$ \\ ${ }^{1}$ School of Pharmacy \& Pharmaceutical Sciences and Trinity Biomedical Sciences Institute, Trinity College Dublin, Dublin 2, \\ Ireland \\ ${ }^{2}$ Department of Clinical Medicine, Aarhus University, 8000 Aarhus C, Denmark \\ Correspondence to: Lorraine O'Driscoll, email: lodrisc@tcd.ie \\ Keywords: metabolic syndrome; obesity; miR-758-3p; CERP/ABCA 1; biomarker \\ Received: July 26, $2017 \quad$ Accepted: January 02, $2018 \quad$ Published: January 24, 2018 \\ Copyright: $O$ 'Neill et al. This is an open-access article distributed under the terms of the Creative Commons Attribution License 3.0 \\ (CC BY 3.0), which permits unrestricted use, distribution, and reproduction in any medium, provided the original author and source \\ are credited.
}

\section{ABSTRACT}

Due to increasing prevalence of obesity, a simple method or methods for the diagnosis of metabolic syndrome are urgently required to reduce the risk of associated cardiovascular disease, diabetes and cancer. This study aimed to identify a miRNA biomarker that may distinguish metabolic syndrome from obesity and to investigate if such a miRNA may have functional relevance for metabolic syndrome.

52 adults with clinical obesity $(n=26)$ or metabolic syndrome $(n=26)$ were recruited. Plasma specimens were procured from all and were randomly designated to discovery and validation cohorts. miRNA discovery profiling was performed, using array technology, on plasma RNA. Validation was performed by quantitative polymerase chain reaction. The functional effect of miR-758-3p on its predicted target, cholesterol efflux regulatory protein/ATP-binding cassette transporter, was investigated using HepG2 liver cells.

Custom miRNA profiling of $\mathbf{2 5}$ miRNAs in the discovery cohort found miR-758$3 p$ to be detected in the obese cohort but undetected in the metabolic syndrome cohort. miR-758-3p was subsequently validated as a potential biomarker for metabolic syndrome by quantitative polymerase chain reaction. Bioinformatics analysis identified cholesterol efflux regulatory protein/ATP-binding cassette transporter as miR-758-3p's predicted target. Specifically, mimicking miR-758-3p in HepG 2 cells suppressed cholesterol efflux regulatory protein/ATP-binding cassette transporter protein expression; conversely, inhibiting miR-758-3p increased cholesterol efflux regulatory protein/ATP-binding cassette transporter protein expression.

miR-758-3p holds potential as a blood-based biomarker for distinguishing progression from obesity to metabolic syndrome and as a driver in controlling cholesterol efflux regulatory protein/ATP-binding cassette transporter expression, indicating it potential role in cholesterol control in metabolic syndrome.

\section{INTRODUCTION}

Metabolic Syndrome (MetS) is an ever-increasing concern worldwide due to the increase in obesity and sedentary lifestyles. It is estimated that one quarter of the adult population has MetS [1]. To date there are three main definitions of MetS, set out by the International Diabetes Federation (IDF), the World Health Organisation (WHO) 
and the National Cholesterol Education Program: Third Adult Treatment Panel (NCEP:ATPIII) [2], that differ in minor details $[3,4]$.

Obesity is fundamental to MetS as it appears to precede the emergence of the other MetS risk factors [5]. MetS culminates from the clustering of component risk factors including obesity [6]. Individuals with MetS are five times more likely to develop Type 2 diabetes (T2DM) and three times more likely to suffer from cardiovascular disease (CVD) [7]. Furthermore, the development of many cancer types is associated with obesity and MetS. A metaanalysis of 38,940 cases of cancer found the presence of MetS to be significantly associated with liver, colorectal, bladder, endometrial, pancreatic, and postmenopausal breast cancers [8]. As the prevalence of MetS is increasing, the prevalence of these conditions is increasing in parallel. Accurate and timely diagnosis of MetS is, therefore, of critical importance to avoid this increased risk.

Despite the increasing prevalence and thus the socioeconomic stresses associated with MetS and its subsequent pathologies, a panel of blood-based miRNA biomarkers for MetS has not yet been established. Mature miRNAs are small $\sim 21$ nucleotide long noncoding single-stranded RNAs that are widely distributed throughout all eukaryotic organisms [9]. miRNAs are encoded in the genome [10] and they bind in an anti-sense manner to complementary mRNA. miRNAs function to increase or decrease gene expression post-transcriptionally [11]. A number of miRNAs have been identified that increase or decrease components of MetS, cholesterol and fatty acid synthesis (e.g. miR-33a/33b, miR-758, miR-204, miR-106b and miR200). Some proteins such as adiponectin [12], leptin [13] and human c-reactive protein $[14,15]$ have been proposed as new biomarkers. However, in reality, in the clinic MetS is diagnosed by assessing insulin resistance, triglyceride levels, high density lipoprotein cholesterol, blood pressure and body-mass index or waist circumference; the latter which some individuals find embarrassing and so avoid being tested. Profiling circulating miRNAs could realistically contribute to a more comprehensive panel of minimally-invasive, blood-based biomarkers for diagnosing MetS. Thus, here we profiled circulating plasma miRNAs from MetS and obese individuals to identify if any miRNAs exist that might distinguish MetS from obesity and that may have diagnostic and functional relevance in the pathophysiology of this condition.

\section{RESULTS}

\section{Participant plasma specimens}

Ninty-nine individuals initially gave informed consent, however 25 withdrew consent prior to screening. A total of 74 were screened, from these 74 there were 11 dropouts (screen failures, 4 problems with IV access, 2 personal reasons, 1 due to disease exacerbation). A total of
63 began the study, however due to technical difficulties no intravenous access was obtained for one of the participants. Fat biopsy specimens were not obtained from 3 participants ( 2 due to bleeding and one did not consent for the biopsy). Furthermore, adipose tissue samples from 2 participants were damaged due to early defrosting (adipose specimens were required for collaborators). A total of 52 particpants completed the study.

\section{RNA yield and characterisation}

A similar yield of RNA was obtained from plasma of individuals with obesity and MetS i.e. $4.85 \pm 0.28 \mu \mathrm{g}$ RNA/ $\mathrm{mL}$ plasma for obese individuals and $4.64 \pm 0.32 \mu \mathrm{g}$ RNA/ $\mathrm{mL}$ plasma for MetS individuals $(\mathrm{p}=0.63$ ) (Figure 1A). Electropherograms from the RNA 6000 Pico kit analysis of the total RNA showed, as expected, that the plasma specimens do not contains ribosomal RNAs (i.e. 18s and $28 \mathrm{~S}$ were not detected). A small peak was detected at 2429secs [s]. (Figure 1B(i)). The subsequent small RNA analysis showed this small peak as RNAs of approximately 10-40 nucleotides (Figure 1B(ii)). It also indicated the percentage of miRNAs detected in the total RNA to not differ significantly $(p=0.59)$ between the RNA isolated from plasma of obese and MetS participants (Figure 1C).

\section{Assessment of miRNAs as potential endogenous control RNAs}

For plasma specimens, conventional "endogenous" control RNAs are often not at a constant level as the assessment is not endogenous per se; but on extracellular RNA. So here we performed a pre-screen of 172 miRNAs per specimen (for $n=20$ specimens; including $n=10$ obese and $n=10$ MetS) in order to seek RNA(s) that may be suitable as controls for this cohort of specimens. From this, miR-128 (average $\mathrm{C}_{\mathrm{T}}=34.2 \pm 0.4$ ), miR-150-5p (average $\mathrm{C}_{\mathrm{T}}=33.4 \pm 0.3$ ), miR-320a (average $\mathrm{C}_{\mathrm{T}}=31.6 \pm 0.3$ ), miR-486-5p (average $\mathrm{C}_{\mathrm{T}}=31 \pm 0.5$ ) and U6 snRNA (average $\mathrm{C}_{\mathrm{T}}=32.7 \pm 0.3$ ) were selected to be included in the custom arrays as potential controls, as all five were detected in all 20 specimens analysed and they were the most uniformly detected miRNAs (average $\mathrm{C}_{\mathrm{T}}=32.6 \pm 0.3$ from all five miRNAs). These five miRNAs were thus included on the custom miRNA array with the 25 miRNAs of interest to test (Table 1). These test miRNAs were selected from a combination of the prescreen and from literature e.g. miR-758-3p was identified as interesting from literature.

\section{miRNAs in plasma specimens from obese and/or MetS individuals}

A cut-off point of $35 \mathrm{C}_{\mathrm{T}}$ was taken, for all miRNAs, where miRNAs detected $\leq 35 \mathrm{C}_{\mathrm{T}}$ were considered as 'present' and those with a $\mathrm{C}_{\mathrm{T}}>35 \mathrm{C}_{\mathrm{T}}$ were considered 'undetected'. This is frequently done for quantitative 
polymerase chain reaction (qPCR) data in order to avoid noise from the qPCR analysis. Of the 25 miRNAs analysed, 18 were detected in plasma from both the obese and MetS cohorts, 2 were detected in plasma from the obese cohort only and one was detected in the plasma of the MetS cohort only. Four miRNAs were undetected in both cohorts (Figure 2).

\section{Identification of potential miRNA biomarkers for MetS - discovery phase}

Following normalisation of the customised test miRNA profiling data to the mean $\mathrm{C}_{\mathrm{T}}$ of miR-150-5p and
miR-486-5p (which were by then found to be the most suitable controls), analysis of dysregulated miRNAs between obese $(n=19)$ and MetS $(n=19)$ was performed. Taking a cut-off point of $\geq 2$-fold increased or decreased in specimens from MetS versus obese individuals, five miRNAs with potential as biomarkers for MetS were identified. Specifically, the quantities of miR-15a-5p (-2.8 \pm 1.4 -fold), miR-106b-5p (-3.1 \pm 1.2 -fold), and miR$200 \mathrm{a}-3 \mathrm{p}(-2.1 \pm 0.4$-fold $)$ were decreased in the MetS cohort and miR-204-5p (2.04 \pm 0.6 -fold) was increased in the MetS cohort (Figure 3A); although statistical significance was not reached due to person-to-person variability. Interestingly, however, miR-758-3p was detected in

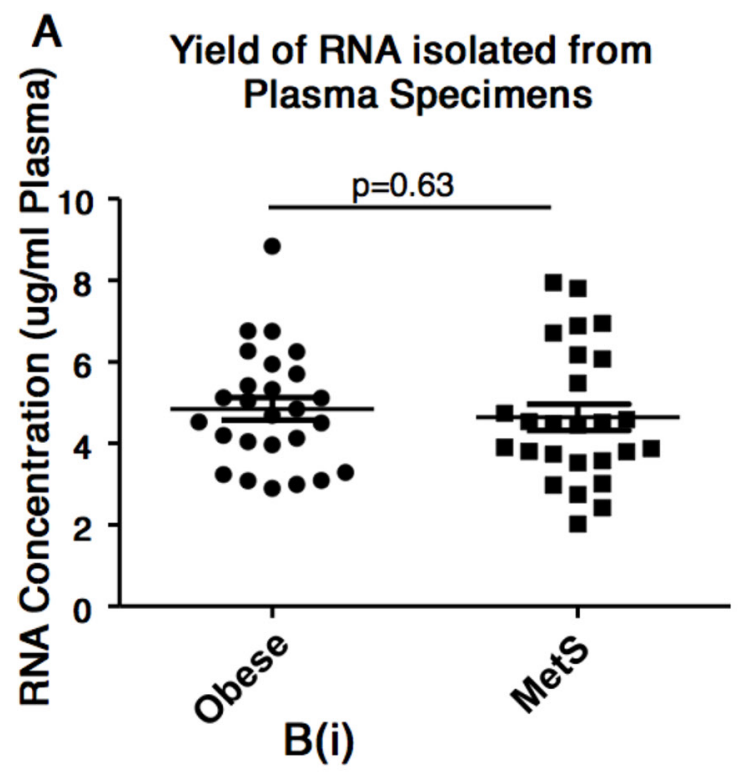

\section{Percentage of miRNAs detected in RNA Isolated from Plasma Specimens}
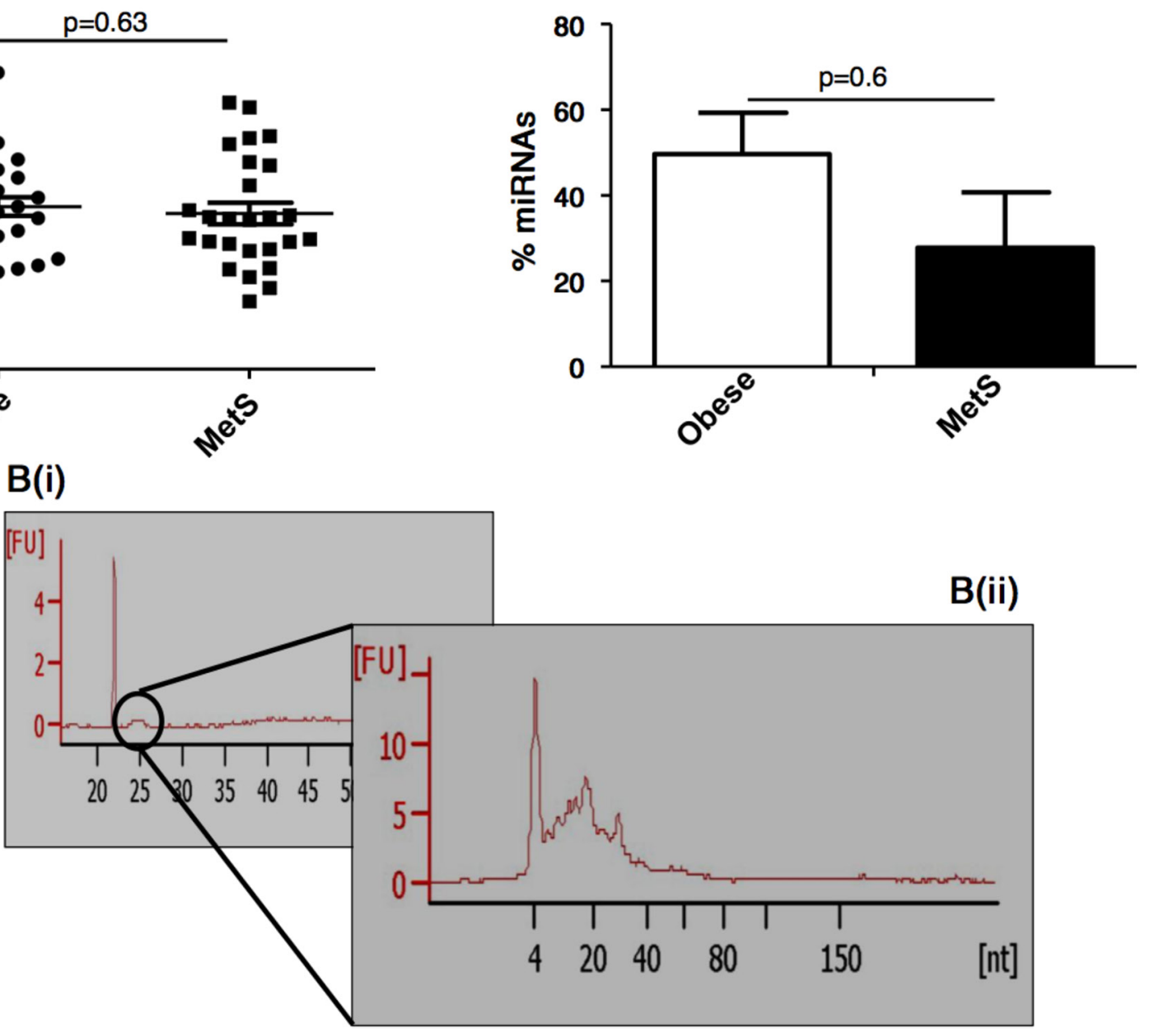

Figure 1: Plasma-based RNA quantification and characterisation (A) Concentration of RNA isolated from plasma specimens, as assessed by NanoDrop ND-1000 spectrophotometer. (B(i)) Electropherograms show the presence of 5S and 5.8S subunits, tRNAs and small RNA fragments (<100bp), but absence of 18S and 28S RNA. (B(ii)) Electropherograms shows the presence of small RNAs. (C) Percentage of miRNAs detected in the total RNA isolated from the plasma of obese and MetS participants. 
Table 1: miRNAs selected for inclusion on customised arrays

\begin{tabular}{|c|c|}
\hline Selected for Profiling & Reason For Selection from Pre-screen \\
\hline $\operatorname{miR}-223-3 p$ & Substantially decreased in MetS vs Obese $(-6.6 \pm 0.9$-fold $)$ \\
\hline $\operatorname{miR}-126-3 p$ & $>2$-fold decreased in MetS vs Obese $(-2.29 \pm 0.89)$ \\
\hline miR-107 & $>2$-fold decreased in MetS vs Obese $(-2.51 \pm 0.69)$ \\
\hline miR-106a-5p & Substantially decreased in MetS vs Obese $(-4.56 \pm 1.16)$ \\
\hline $\operatorname{miR}-29 a-3 p$ & $>2$-fold decreased in MetS vs Obese $(-2.86 \pm 0.98$-fold $)$ \\
\hline $\operatorname{miR}-122-5 p$ & $>2$-fold increased in MetS vs obese $(2.86 \pm 0.94$-fold $)$ \\
\hline $\operatorname{miR}-103 a-3 p$ & Substantially decreased in MetS vs Obese $(-7.31 \pm 1.2)$ \\
\hline $\operatorname{miR}-92 a-3 p$ & $>2$-fold decreased in MetS vs Obese $(-2.43 \pm 0.80$-fold $)$ \\
\hline $\operatorname{miR}-106 b-5 p$ & Substantially decreased in MetS vs Obese $(-7.7 \pm 0.95$-fold $)$ \\
\hline let-7d-5p & Substantially decreased in MetS vs Obese (-11.20 \pm 0.94 -fold) \\
\hline let-7f-5p & $>2$-fold decreased in MetS vs Obese $(-3.5 \pm 0.64$-fold $)$ \\
\hline $\operatorname{miR}-26 b-5 p$ & Substantially decreased in MetS vs Obese $(-5.19 \pm 0.84)$ \\
\hline $\operatorname{miR}-23 a-3 p$ & $>2$-fold decreased in MetS vs Obese $(-3.76 \pm 0.79)$ \\
\hline $\operatorname{miR}-23 b-3 p$ & $>2$-fold decreased in MetS vs Obese $(-2.76 \pm 0.7)$ \\
\hline $\operatorname{miR}-15 a-5 p$ & $>2$-fold decreased in MetS vs Obese $(-3.4 \pm 1.03)$ \\
\hline $\operatorname{miR}-130 a-3 p$ & Substantially decreased in MetS vs Obese $(-7.31 \pm 1.2)$ \\
\hline $\operatorname{miR}-200 c-3 p$ & $>2$-fold increased in MetS vs Obese $(3.72 \pm 0.8)$ \\
\hline $\operatorname{miR}-423-5 p$ & Increased in MetS vs Obese (1.6 \pm 0.7 -fold $)$ \\
\hline $\operatorname{miR}-200 a-3 p$ & Cited as [31] regulating glucose metabolism \\
\hline $\operatorname{miR}-29 b-3 p$ & Cited as [32] regulating glucose metabolism \\
\hline $\operatorname{miR}-204-5 p$ & Cited as [33] regulating adipocyte differentiation \\
\hline $\begin{array}{l}\operatorname{miR}-33 a-3 p, \text { miR-33b-3p, miR- } \\
33 b-5 p\end{array}$ & Cited as [34] regulating lipid metabolism. \\
\hline $\operatorname{miR}-758-3 p$ & Cited as [35] regulating cholesterol metabolism \\
\hline Controls RNAs & miR-128, miR-486-5p, miR-320a, miR-150-5p, U6 snRNA \\
\hline
\end{tabular}

all plasma specimens from those with obesity, and was undetected in all plasma specimens from those with MetS (Figure 3B).

\section{qPCR validation of results arising from discovery phase - validation phase}

Individual qPCR assays for miR-15a-5p, miR106b-5p, miR-200a-3p, miR-204-5p and miR-758-3p in the validation cohort $(n=14,7=$ obese; $7=$ MetS $)$ confirmed that four of these five miRNAs were not at significantly different amounts in the MetS cohort compared to the obese cohort. However, miR-758-3p was, again, detected in the obese cohort and undetected in the MetS cohort, validating this result from the discovery phase and suggesting that miR-758-3p may be a biomarker for MetS (Figure 3C).

\section{Investigation of CERP/ABCA1 as a target of miR-758-3p}

Plasma miR-758-3p demonstrated clinical relevance as a biomarker for progression of obesity to MetS, so our subsequent analysis was to investigate if miR-758-3p can target and control expression of cholesterol efflux regulatory protein/ATP-binding cassette transporter (CERP/ABCA1); its predicted target. As cholesterol efflux occurs in the liver, we selected HepG2 liver cells for further analysis. Here, we used an LXR agonist T0901317 to induce expression ABCA1 in the HepG2 cell line. Inhibition of miR-758-3p resulted in a significant (95\% CI: 0.3074-49.70, $\mathrm{p}=0.0483$ ) increase in the CERP/ ABCA1 expression in the HepG2 cell line compared to CERP/ABCA1 expression in negative control inhibitor transfected cells (Figure 4A). Conversely, mimicked 
expression of miR-758-3p resulted in a significant $(95 \%$ CI: $3.467-58.98, p=0.0354)$ decrease in the levels of CERP/ABCA1 compared to negative control mimic transfected cells (Figure 4B). This supports miR-758-3p targeting and controlling $\mathrm{CERP} / \mathrm{ABCA} 1$ expression in liver cells.

\section{DISCUSSION}

The role of miRNAs and their potential as biomarkers for diseases such as cancer has long been investigated by ourselves [16-18] and others [19, 20]. Here, we progressed to investigate the potential use of blood-based miRNAs as biomarkers for metabolic syndrome (MetS) and the putative ability of miRNAs in the peripheral circulation to discriminate progression from obesity to MetS [3]. To the best of our knowledge, this is the first examination of the differences in bloodbased miRNAs in MetS individuals compared to obese individuals.

Our study was designed in such a way as to only include blood plasma specimens from individuals who were being selected to subsequently take part in a randomised, parallel controlled, double-blinded, dietary intervention study (DairyHealth [21]). This study design was to ensure comprehensive prior clinical assessment of the individuals, so as to accurately characterise them as clinically obese or MetS; to fully adhere to the inclusion and exclusions criteria described here; to only procure blood specimens from individuals in a fasting state and in the morning; and to process all specimens using standard operation procedures performed by appropriately trained

\section{Detected in neither obesity or MetS}

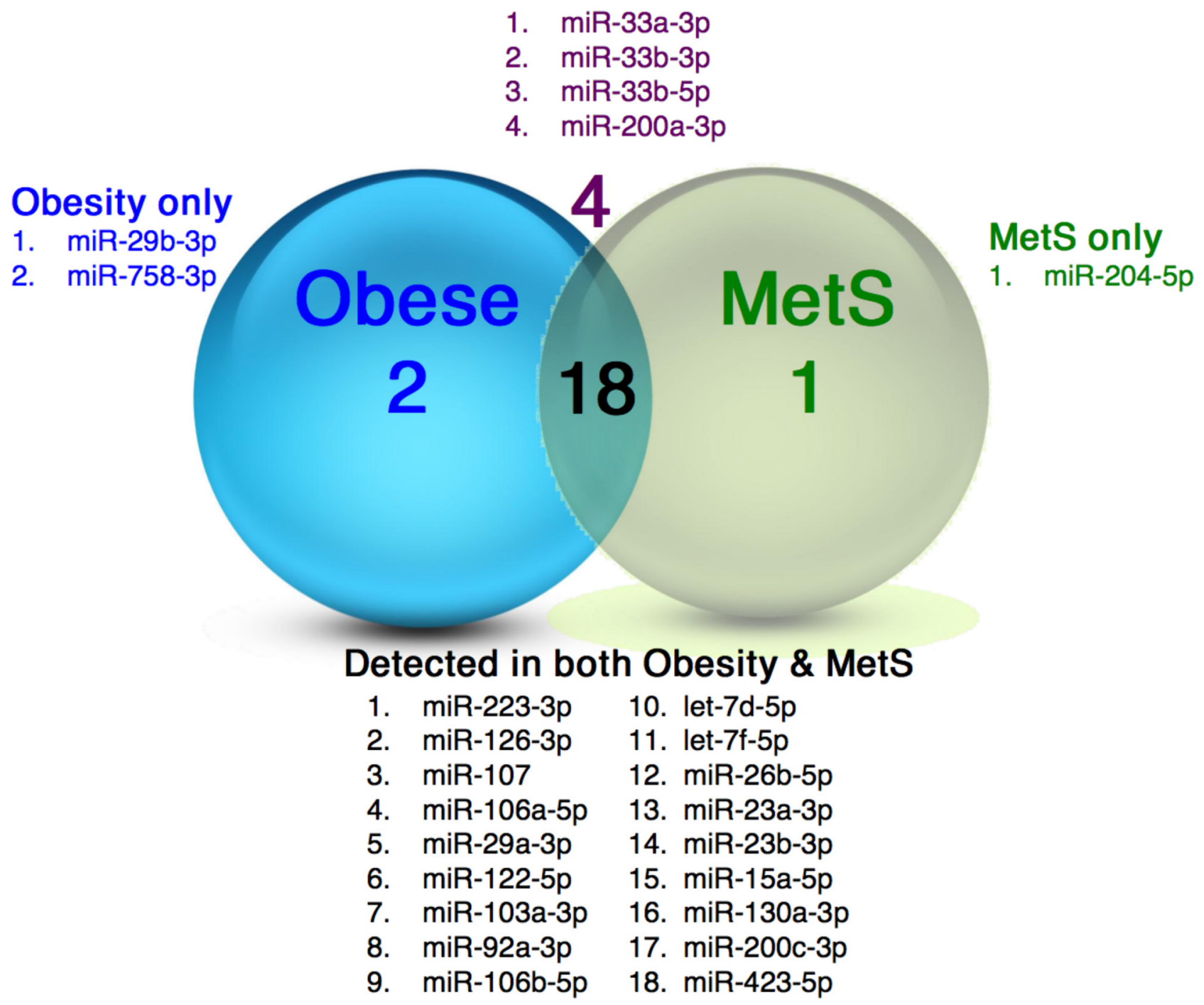

Figure 2: miRNAs in plasma specimens from obese and MetS participants. Illustration of the distribution of miRNAs detected in plasma from the obese and MetS individuals. 
personnel. The reason for applying this level of stringency was to avoid, in as far as at all possible, noise in data and differences in results that can occur in biomarker studies that are not designed prospectively. As a simple example, if participants donated blood specimens at various times throughout the day, some before and some after meals, there are increased chances of differences in circulating miRNAs being at least partially due to effects of these variables and not more specifically due to biological differences between obesity and MetS. Additionally, these plasma specimens are limited in volume (as they are part of an intervention study), as many other baseline parameters had to be assessed $[21,22]$. Thus, we elected to perform a pre-screen on a limited number $(n=20)$ of specimens. We also considered key miRNAs from literature mining. This was to inform a rationally-designed focussed analysis of 25 miRNAs, rather than what is sometime described as a "fishing trip" of many hundreds of miRNAs but on fewer specimens. For example miR-204-5p was found to be significantly increased in the MetS cohort (10 specimens) compared to the obese cohort (10 specimens) following the pre-screen. This was interesting as miR-204-5p has been shown to be increase during adipogenesis [4].

Of note, efforts were also made to identify control miRNAs against which to normalise our data, as we [17] and others working in the field of blood-based miRNAs have previously reported. Serum/plasma studies do not involve miRNAs that are actually intracellular/endogenous and to date no single blood-based miRNA that suits all study comparisons has been identified. However, in these particular conditions i.e. obesity and MetS, we found the mean levels of a number of miRNAs most suitable (rather than a single miRNA) as control miRNAs for normalising our data. Furthermore, with plasma specimens procured from 52 individuals (26 with MetS, 26 with obesity) one option we had was to perform only a "discovery" study i.e. only investigate differential miRNA detection, with the hope of attempting an initial validation in the future. However, when designing the study -with all clinicians, statisticians and scientists in agreement- a more meaningful

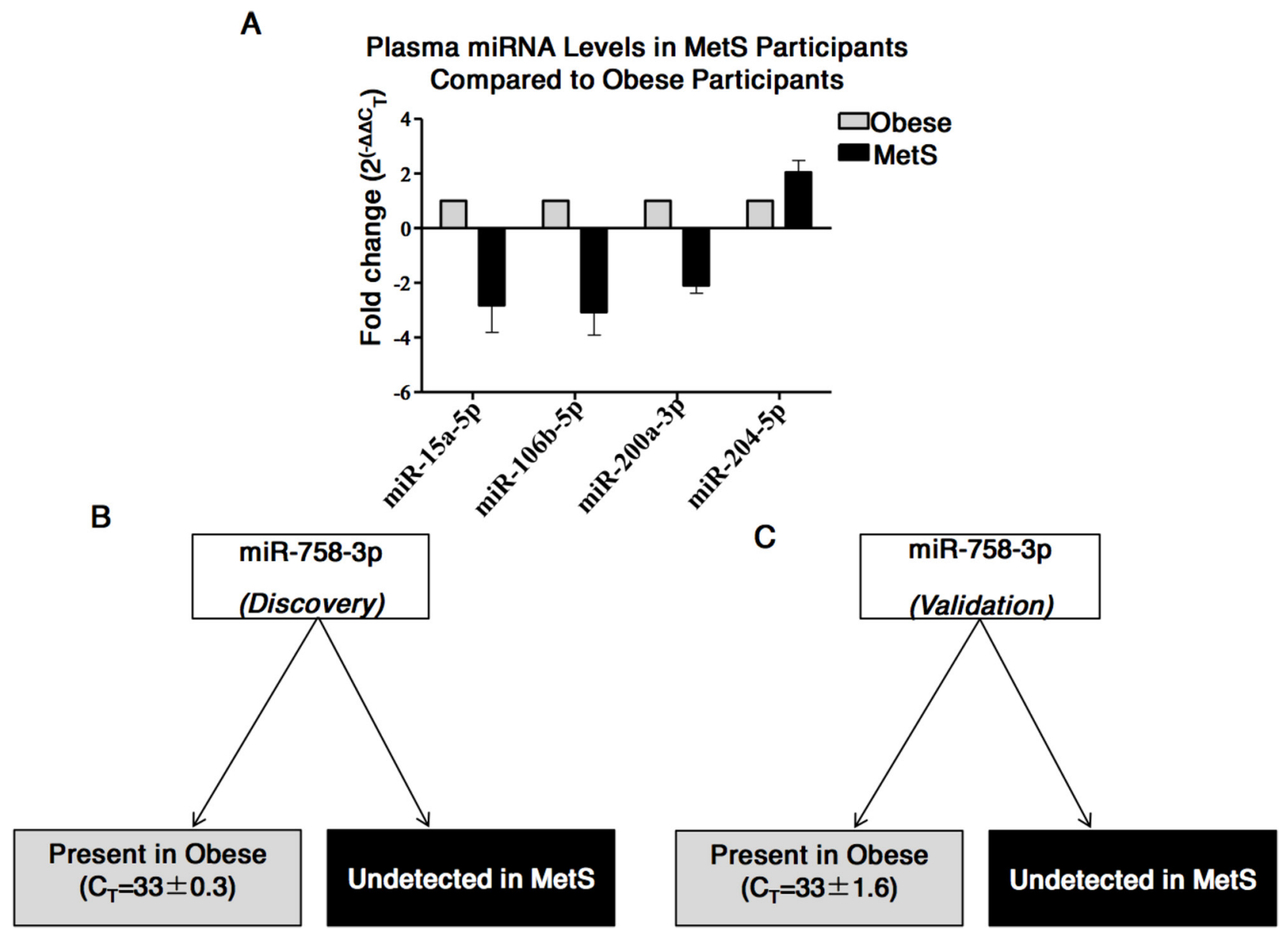

Figure 3: Differences in miRNAs in plasma from MetS compared to Obese participants. (A) Five miRNAs found to have $\geq 2$-fold difference in quantities in the plasma from MetS compared to obesity. (B) miR-758-3p was detected in plasma of obese participants and undetected in the plasma of MetS participants during the discovery stage. (C) miR-758-3p detected in plasma of obese participants and undetected in the plasma of MetS participants during the validation stage. 
and useful study, for ourselves and others to build upon, would include a discovery and a validation stage.

Using this approach of a random discovery cohort and validation cohort, we identified miR-758-3p as a potential biomarker for discriminating MetS from obesity i.e. miR-758-3p was undetected in plasma from individuals in the MetS cohort but was detected in plasma from all individuals in the obese cohort. We believe that further studies to independently validate these finding are now warranted.

To understand if the loss of miR-758-3p in progression from obesity to MetS may reflect pathophysiological relevance, we then investigated the potential targets of miR-758-3p using miRNA-target predictor miR-walk, miR-Walk collates predictions from DIANAmT, miRanda, miRDB, miR-Walk, RNAhybrid, PICTAR4, PICTAR5, PITA, RNA22 and Targetscan.
Here, cholesterol efflux regulatory protein/ATP-binding cassette transporter A1 (i.e. CERP/ABCA1) was predicted as the target of miR-758-3p. CERP/ABCA1 is a transmembrane transporter [23] that, for many years, has been well established as a key mediator of cholesterol homeostasis, facilitating cellular free cholesterol efflux to an extracellular acceptor (apolipoprotein AI, apoA-I) to form nascent high density lipoprotein $\operatorname{HDL}[24,25]$. Approximately $70 \%$ of nascent HDL is generated in the liver when Apo-AI, which is generated by hepatocytes, interacts with hepatic CERP/ABCA1 [26]. Interestingly, in a recent study of CERP/ABCA1 cholesterol efflux in serum from people with MetS and healthy controls, MetS patients were found to present significantly higher CERP/ ABCA1 mediated cholesterol efflux than their healthy counterparts. To accept cholesterol from cholesterol-loaded BHK cells expressing either ABCA1 or ABCG1 [27].

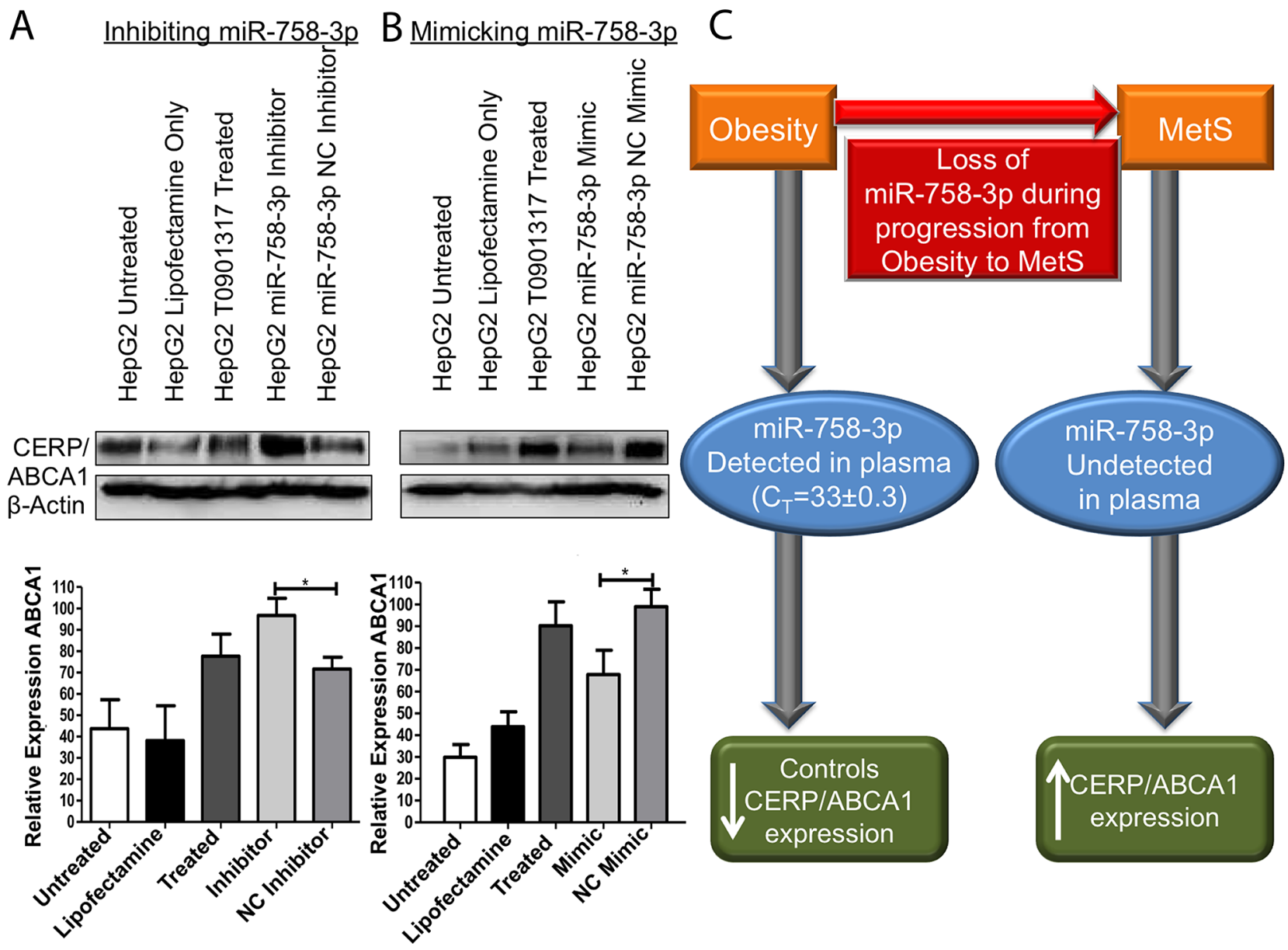

Figure 4: miR-758-3p can control expression of CERP/ABCA1. (A) Inhibition of miR-758-3p in HepG2 cells treated with T0901317 (to induce CERP/ABCA1 expression) resulted in a significant $(\mathrm{p}<0.05)$ increase in CERP/ABCA1 protein expression compared to ABCA1 levels in negative control (NC) inhibitor transfected cells. (B) Conversely, mimicked expression of miR-758-3p in HepG2 cells treated with T0901317 caused a significant reduction in CERP/ABCA1 expression compared to NC mimic transfected cells. Results are shown as representative immunoblots and graphs of densitometry from all immunoblots i.e. $\mathrm{n}=3 \pm \mathrm{SEM}{ }^{*} \mathrm{p}<0.05,{ }^{* *} \mathrm{p}<0.01,{ }^{* *} \mathrm{p}<0.001$. (C) Proposed model of miR-758-3p, which was detected in plasma in obesity and can control CERP/ABCA1 expression, while in MetS the loss of miR-758-3p is associated with increased expression of CERP/ABCA1. Immunoblots were uniformly altered using brightness and contract settings in Powerpoint. 
Table 2: Participant characteristics

\begin{tabular}{lcc}
\hline Participant Characteristics & Male & Female \\
\hline Age $(\mathrm{yrs} \pm$ SEM) & $56.7 \pm 2.03$ & $56.3 \pm 2.14$ \\
MetS (n) & 12 & 14 \\
Obese (n) & 13 & 13 \\
BMI $\left(\mathrm{kg} / \mathrm{m}^{2}\right) \pm$ SEM & $28.8 \pm 0.51$ & $30.2 \pm 4.4$ \\
Anti-hypertensive treatment & $6(24 \%)$ & $3(11.1 \%)$ \\
Cholesterol lowering treatment & $5(20 \%)$ & $6(22.2 \%)$ \\
\hline
\end{tabular}

As CERP/ABCA1 has been shown to be responsible for inducing formation of HDL in the liver, to confirm our hypothesis that loss of miR-758-3p may reflect pathophysiological relevance, we selected the liver cell line HepG2 for subsequent functional studies. Using both mimics and inhibitors, in keeping with the work of Ramirez et al. [28] and Lucero et al. [27], we confirmed that loss of miR-758-3p (as we observed in MetS) resulted in increased expression of CERP/ABCA1 on the liver cells. This may be the mechanism responsible for the significantly higher CERP/ABCA1 mediated cholesterol efflux reported in MetS [27]. Conversely, we found miR$758-3 p$ mimics to have the opposite effect on CERP/ ABCA1 i.e. to reduce CERP/ABCA1 expression, which was made more difficult to achieve given that the use of an LXR agonist to induce ABCA1 would reduce the efficacy of the miR-758-3p mimic. Taken together, this suggests that modulating miR-758-3p may have therapeutic relevance in conditions contributed to by too little or too much miR-758-3p expression; such as MetS.

In conclusion, our study shows for the first time that miR-758-3p holds potential as a minimally-invasive blood-based biomarker for aiding the diagnosis of MetS, by distinguishing MetS individuals from those with clinical obesity. Efforts towards progressing miR-758-3p to the clinic for use as a diagnostic biomarker would, of course, involve the validation of the results shown here in independent cohorts of participants. Considering the potential pathophysiological relevance of this loss of miR$758-3 p$ in the progression of obesity to MetS, we also provide functional evidence that miR-758-3p targets CERP/ ABCA1 in liver cells, in keeping with the reported higher CERP/ABCA1 mediated cholesterol efflux in MetS (in that case, compared to healthy controls). Identification of this miRNA as a potential blood-based biomarker for MetS and possibly also causally involved in these events- may help address an unmet need, as the societal and economic burden of obesity progressing to MetS and the subsequent increased risk of CVD, T2DM and cancer is substantial. Further independent assessment of miR-758-3p and its control of CERP/ABCA1 - both as a diagnostic biomarker and a therapeutic intervention- is now warranted. It would also be very interesting to establish if the miRNAs detected are associated with RNA-binding protein in the sera or if they are associated with extracellular vesicles.

\section{MATERIALS AND METHODS}

\section{Study design and participant plasma specimens}

63 individuals were recruited to DairyHealth (NCT01472666) in the Department of Endocrinology and Internal Medicine Aarhus University, Denmark between October 2011 and December 2012 through local newspapers and electronic advertisement [21]. The number of participants needed to complete the study and achieve a statistical power of $80 \%$ was calculated to be $52(\alpha<0.05, \beta=0.80)$. Fifty-two individuals including 26 individuals who were diagnosed with MetS according to the IDF definition (female: $n=14,59.5 \pm 3.2$ yrs; male: $\mathrm{n}=12,58.6 \pm 3.8 \mathrm{yrs})$ and 26 individuals who were clinically obese (female: $\mathrm{n}=13,47.1 \pm 5.2$ yrs; male: $\mathrm{n}=13,61.5 \pm 2.9$ yrs) completed the study (see Table 2 for participant characteristics). Participants were screened on the basis of their medical history and a physical examination. Inclusion criteria included $\geq 18$ years; abdominal circumference of $\geq 90 \mathrm{~cm}$ (males) and $\geq 80 \mathrm{~cm}$ (females); weight stable $( \pm 5 \mathrm{~kg}$ ) for at least 3 months prior to inclusion. Exclusion criteria included T2DM; severe cardiovascular, renal, endocrine or psychiatric disease; drug abuse; pregnancy or lactation; and blood donation was prohibited for 3 months prior to inclusion.

Upon arrival at the clinic in the morning, individuals were fasting and a blood specimen was procured, plasma was isolated and stored at $-80^{\circ} \mathrm{C}$ for subsequent analysis. The study was designed to include a two-stage analysis of miRNAs present in the plasma i.e. a discovery stage, using a customised array for a total of 38 specimens, including specimens from 19 individuals with obesity and 19 individuals with MetS and being mindful to balance male and female participants within these sub-cohorts, but otherwise selected randomly. This was followed by a validation stage, by qPCR, for the remaining 14 specimens $(\mathrm{n}=7$ obesity; $\mathrm{n}=7$ MetS). The study protocol was performed in accordance with the Helsinki Declaration of 1975 as revised in 1983 and was approved by the 
Central Denmark Region Committees on Health Research Ethics. The study was registered with clinicaltrials.gov as NCT01472666. Additionally, after receiving written and oral information, all participants gave their written informed consent before participating in the study.

\section{RNA extraction and characterisation}

RNA was isolated from $250 \mu \mathrm{L}$ of each of the plasma specimens. Specifically, RNA was extracted using TriReagent (Sigma-Aldrich, T9424) using a modified procedure that we had previously reported [29]. In brief, $200 \mu \mathrm{L}$ chloroform (Sigma-Aldrich, C424) was added to each specimen, followed by a $15 \mathrm{~min}$ incubation, centrifugation and retention of the aqueous phase. Glycogen (Sigma, G8751) (final concentration 120 $\mu \mathrm{g} / \mathrm{mL}$ ) was used as a carrier and $500 \mu \mathrm{L}$ isopropanol (Sigma, 19516) was added to precipitate out the RNA. RNA quantity was subsequently assessed using a NanoDrop ND-1000 (Labtech International, Uckfield, England) and Agilent Pico 6000 and Small RNA kits (Agilent Technologies, CA, USA) as per manufacturers' instructions.

\section{miRNA profiling}

Prior to initiating the discovery phase, efforts were made to identify one or more controls that may be unchanged in all specimens. This involved using Exiqon's Focus Panel (Exiqon, 203843) consisting of 172 miRNAs and 7 plate control RNAs for 20 specimens. A customer-designed Exiqon pick-and-mix panel was subsequently generated as the array platform for profiling (Exiqon, 203815) for use with the ABI-7900HT Sequence Detection System (Applied Biosystems, Foster City, CA, USA). Twenty-five miRNAs and a further five sequences identified as potential controls, selected from a combination of the Focus Panel and informed by published literature (Table 1), were placed on the customised arrays along with UniSp3IPC and UniSp6 technical control

\section{Reverse transcription (cDNA synthesis) discovery miRNA profiling}

Reverse transcription (RT) of miRNAs to cDNA was performed according to the Exiqon miCURY LNA ${ }^{\mathrm{TM}}$ Universal RT miRNA PCR instruction manual for serum/ plasma and other biofluids samples. Briefly, RNA (suspended in $1.6 \mu \mathrm{L}$ of nuclease-free water) for each specimen was added to $2 \mu \mathrm{L}$ of $5 \mathrm{X}$ reaction buffer, $1 \mu \mathrm{L}$ of enzyme mix, $4.9 \mu \mathrm{L}$ of nuclease-free water and 0.5 $\mu \mathrm{L}$ of UniSp6 spike-in. cDNA was synthesised using the Applied Biosystems Veriti 96 well thermal cycler (Applied Biosystems, Foster City, CA, USA) as follows: incubate at $42^{\circ} \mathrm{C}$ for $60 \mathrm{mins}$, heat-inactivate the reverse transcriptase at $95^{\circ} \mathrm{C}$ for 5 mins and cool to $4^{\circ} \mathrm{C}$.

\section{qPCR}

qPCR was performed also according to the Exiqon miCURY LNA ${ }^{\mathrm{TM}}$ Universal RT miRNA PCR instruction manual for serum/plasma and other biofluid samples. As the pre-screen showed miR-486-5p and miR-150-5p quantities do not differ significantly between specimens, these were considered as controls. $\mathrm{C}_{\mathrm{T}}$ values for other miRNAs were normalised to the mean quantity of miR486-5p plus miR-150-5p. qPCR was performed using the ABI-7900HT Sequence Detection System with the conditions as recommended by Exiqon i.e. hold at $95^{\circ} \mathrm{C}$ for 10 mins; qPCR stage at $95^{\circ} \mathrm{C}$ for $10 \mathrm{secs}$ and $60^{\circ} \mathrm{C}$ for $1 \mathrm{~min}$, by 40 cycles and melt stage at $95^{\circ} \mathrm{C}$ for $15 \mathrm{secs}$, $60^{\circ} \mathrm{C}$ for $1 \mathrm{~min}, 95^{\circ} \mathrm{C}$ for $15 \mathrm{secs}$.

\section{Validation of potential biomarkers for MetS}

Subsequent efforts to validate 5 miRNAs selected from the discovery stage were performed using sequencespecific primers i.e. miR-15a-5p (Exiqon, 204066), miR-106b-5p (Exiqon, 205884), miR-200a-3p (Exiqon, 204707), miR-204-5p (Exiqon, 205708) and miR-758-3p (Exiqon, 204353). Again, miR-486-5p (Exiqon, 204001) and miR-150-5p (Exiqon, 204660) were included as controls, with UniSp6 as technical control.

\section{Data analysis}

ABI SDS v2.4 software was used to obtain $\mathrm{C}_{\mathrm{T}}$ values. The raw data was exported using SDS software and UnSP3IPC was used to calibrate for differences in plate runs using the equation: Specimen $\mathrm{C}_{\mathrm{T}}$ - ((average of UniSP3IPC for the plate)-(average of UniSp3IPC for all plates)). UniSp6 confirmed the RT and PCR reactions were successful; therefore any miRNAs not detected in the study was not due to technical error. Fold changes of miRNAs in MetS specimens versus obese specimens were subsequently determined by the $\Delta \Delta \mathrm{C}_{\mathrm{T}}$ method.

\section{Bioinformatics analysis}

miR-Walk, collating data from a number of prediction softwares, including DIANAmT, miRanda, miRDB, miRWalk, RNAhybrid, PICTAR4, PICTAR5, PITA, RNA22 and Targetscan, was used to identify potential targets of miRNAs. From this analysis, the cholesterol efflux regulatory protein/ATP-binding cassette transporter (CERP/ ABCA1) was predicted as a miR-758-3p target.

\section{Cell culture}

HepG2 (human liver carcinoma) [30] cells were obtained from ATCC. Cells were authenticated using short tandem repeat profiling (May, 2016) and cultured in Eagle's MEM (Sigma-Aldrich, M2279) containing 10\% fetal bovine serum (Gibco, 10270106), 2mM L-Glutamine 
(Sigma-Aldrich, I0516) and $1 \mathrm{X}$ non-essential amino acids (Sigma-Aldrich, $\mathrm{M} 7145$ ) at $37^{\circ} \mathrm{C}$ with $5 \% \mathrm{CO}_{2}$.

\section{miRNA inhibition/mimic manipulation in HepG2 cells}

HepG2 cells seeded at $4 \times 10^{5}$ cells/well in a 6-well plate were transfected, using lipofectamine 2000 (Invitrogen, 11668-027), with miR-758-3p inhibitor (Dharmacon, 4100323-021), miRNA inhibitor negative control (Dharmacon, 12610636), miR-758-3p mimic (Dharmacon, 15475264) or miRNA mimic negative control (Dharmacon, 11587170); each at a final concentration of $40 \mathrm{nM} .24$ hrs post-transfection, cells were treated with LXR agonist T0901317 (Sigma-Aldrich, T2320) to induce CERP/ABCA1 expression. 24 hours later cells were harvested for protein isolation and immunoblotting.

\section{Immunoblotting}

Total protein $(50 \mu \mathrm{g})$ was resolved on $7 \%$ SDSPAGE and transferred to PVDF membranes (BioRad Laboratories, 162-0177). Primary antibodies to CERP/ABCA1 (Abcam, 18180, 1:200, mouse monoclonal antibody) and $\beta$-actin (Sigma-Aldrich, A5316, 1:1000, mouse monoclonal antibody) were used. Following incubation with the appropriate antimouse horseradish peroxidase-conjugated secondary antibodies (Cell Signalling, 7076s, 1:1000, horse antimouse $\mathrm{IgG}$ antibody), immunoblots were developed using chemiluminescence (Thermo Fisher, 34080) and detected on a Chemidoc exposure system (Bio-Rad Laboratories, Hercules, CA, USA). Densitometry was performed using NIH ImageJ software and CERP/ABCA1 was normalised to the loading control $\beta$-actin.

\section{Statistical analysis}

Statistical analysis was performed in Excel. Fold changes between miRNAs detected in the MetS cohort compared to miRNAs detected in the obese cohort were calculated using the $\Delta \Delta \mathrm{C}_{\mathrm{T}}$ method. Specifically, $\Delta \mathrm{C}_{\mathrm{T}}=$ Each miRNA $C_{T}$ value-average $C_{T}$ value of control miRNAs; $\Delta \Delta \mathrm{C}_{\mathrm{T}}=$ miRNA $\mathrm{C}_{\mathrm{T}}$ value in MetS cohort-miRNA in obese cohort $\mathrm{C}_{\mathrm{T}}$ value; fold change $=\Delta \Delta \mathrm{C}_{\mathrm{T}}$ plugged into formula $2^{(-\Delta \Delta C)}{ }_{T}$. P-values were generated using Student's $T$-tests, with $\mathrm{p}<0.05$ considered as statistically significant. GraphPad Prism 5.0 was used for graph generation (Graph Pad Software Inc, La Jolla, CA, USA). Analysis of the raw data was performed both prior to and following controlling for clinical data available for all participants (Table 2). However, no difference prior to and after controlling for these parameters was observed.

\section{Abbreviations}

CERP/ABCA1: cholesterol efflux regulatory protein/ ATP-binding cassette transporter; CT: cycle threshold; MetS: metabolic syndrome; IDF: International Diabetes Federation; WHO: World Health Organisation; NCEP:ATPIII: National Cholesterol Education Program: Third Adult Treatment Panel; T2DM: Type 2 Diabetes Mellitus; CVD: cardiovascular disease; Apo-A1: Apolipoprotein A1.

\section{Author contributions}

LOD, SON, MBL, SG, KH contributed to the idea and design of the study. SON, under the supervision of LOD acquired the laboratory-based data. MBL, with SG and $\mathrm{KH}$, acquired the associated participants' data. SON, under the supervision of LOD, analysed the data. LOD, SON, MBL, SG, KH were all involved in interpreting the data. SON and LOD drafted the article and revised it critically for important intellectual content, with intellectual input also from MBL, SG and KH. All authors read and approved the final manuscript.

\section{ACKNOWLEDGMENTS}

We thank Dr. Sweta Rani, Post-Doctoral Fellow, for assistance with training Sadhbh O'Neill at the start of her $\mathrm{PhD}$ project.

\section{CONFLICTS OF INTEREST}

All authors declare that there is no duality of interest associated with their contribution to this manuscript.

\section{FUNDING}

This work was supported by the Danish Council for Strategic Research (Innovation Fund Denmark), and the Higher Education Authority's PRTLI Cycle 5 funding of TBSI. Funding sources had no involvement in study design, data generation or analysis, writing of manuscript or decision to submit for publication.

\section{REFERENCES}

1. Dunstan DW, Zimmet PZ, Welborn TA, De Courten MP, Cameron AJ, Sicree RA, Dwyer T, Colagiuri S, Jolley D, Knuiman M, Atkins R, Shaw JE. The rising prevalence of diabetes and impaired glucose tolerance: the australian diabetes, obesity and lifestyle study. Diabetes care. 2002; 25:829-34.

2. Alberti KG, Zimmet P. The metabolic syndrome: time to reflect. Current diabetes reports. 2006; 6:259-61.

3. O’Neill S, O’Driscoll L. Metabolic syndrome: a closer look at the growing epidemic and its associated pathologies. Obesity reviews. 2015; 16:1-12.

4. O'Neill S, Bohl M, Gregersen S, Hermansen K, O'Driscoll L. Blood-based biomarkers for metabolic syndrome. Trends in endocrinology and metabolism. 2016; 27:363-74. 
5. Deedwania PC, Gupta R. Management issues in the metabolic syndrome. The Journal of the Association of Physicians of India. 2006; 54:797-810.

6. Cornier MA, Dabelea D, Hernandez TL, Lindstrom RC, Steig AJ, Stob NR, Van Pelt RE, Wang H, Eckel RH. The metabolic syndrome. Endocrine reviews. 2008; 29:777-822.

7. Sookoian S, Pirola C. Metabolic syndrome: from the genetics to the pathophysiology. Curr Hypertens Rep. 2011; 13:149-57.

8. Esposito K, Chiodini P, Colao A, Lenzi A, Giugliano D. Metabolic syndrome and risk of cancer: a systematic review and meta-analysis. Diabetes care. 2012; 35:2402-11.

9. Jensen S, Lamy P, Rasmussen M, Ostenfeld M, Dyrskjot L, Orntoft T, Andersen C. Evaluation of two commercial global miRNA expression profiling platforms for detection of less abundant miRNAs. BMC Genomics. 2011; 12:435-47.

10. Lewis AP, Jopling CL. Regulation and biological function of the liver-specific miR-122. Biochemical Society transactions. 2010; 38:1553-7.

11. Vasudevan S. Posttranscriptional upregulation by microRNAs. Wiley interdisciplinary reviews RNA. 2012; 3:311-30.

12. Kishida K, Funahashi T, Shimomura I. Adiponectin as a routine clinical biomarker. Best practice \& research Clinical endocrinology \& metabolism. 2014; 28:119-30.

13. Ahonen TM, Saltevo JT, Kautiainen HJ, Kumpusalo EA, Vanhala MJ. The association of adiponectin and low-grade inflammation with the course of metabolic syndrome. Nutr Metab Cardiovasc Dis. 2012; 22:285-91.

14. Devaraj S, Singh U, Jialal I. Human C-reactive protein and the metabolic syndrome. Current opinion in lipidology. 2009; 20:182-9.

15. Huffman FG, Gomez GP, Zarini GG. Metabolic syndrome and high-sensitivity C-reactive protein in Cubans. Ethnicity \& disease. 2009; 19:115-20.

16. Friel AM, Corcoran C, Crown J, O’Driscoll L. Relevance of circulating tumor cells, extracellular nucleic acids, and exosomes in breast cancer. Breast cancer research and treatment. 2010; 123:613-25.

17. Rani S, Gately K, Crown J, O’Byrne K, O’Driscoll L. Global analysis of serum microRNAs as potential biomarkers for lung adenocarcinoma. Cancer biology \& therapy. 2013; 14:1104-12.

18. Corcoran C, Rani S, O'Driscoll L. miR-34a is an intracellular and exosomal predictive biomarker for response to docetaxel with clinical relevance to prostate cancer progression. The Prostate. 2014; 74:1320-34.

19. Hayes J, Peruzzi PP, Lawler S. MicroRNAs in cancer: biomarkers, functions and therapy. Trends in molecular medicine. 2014; 20:460-9.

20. Peng $\mathrm{Y}$, Croce CM. The role of MicroRNAs in human cancer. Signal Transduction And Targeted Therapy. 2016; 1:15004.

21. Bohl M, Bjørnshave A, Rasmussen KV, Schioldan AG, Amer B, Larsen MK, Dalsgaard TK, Holst JJ, Herrmann A, O’Neill S, O’Driscoll L, Afman L, Jensen E, et al.
Dairy proteins, dairy lipids, and postprandial lipemia in persons with abdominal obesity (DairyHealth): a 12-wk, randomized, parallel-controlled, double-blinded, diet intervention study. The American journal of clinical nutrition. 2015; 101:870-8.

22. Bohl M, Bjornshave A, Gregersen S, Hermansen K. Whey and casein proteins and medium-chain saturated fatty acids from milk do not increase low-grade inflammation in abdominally obese adults. The review of diabetic studies. 2016; 13:148-157.

23. Westerterp M, Bochem AE, Yvan-Charvet L, Murphy AJ, Wang N, Tall AR. ATP-binding cassette transporters, atherosclerosis, and inflammation. Circ Res. 2014; 114:157-70.

24. Wang S, Smith JD. ABCA1 and nascent HDL biogenesis. BioFactors (Oxford, England). 2014; 40:547-54.

25. Wang N, Silver DL, Thiele C, Tall AR. ATP-binding cassette transporter A1 (ABCA1) functions as a cholesterol efflux regulatory protein. The Journal of biological chemistry. $2001 ; 276: 23742-7$.

26. Vaisman BL, Demosky SJ, Stonik JA, Ghias M, Knapper CL, Sampson ML, Dai C, Levine SJ, Remaley AT. Endothelial expression of human ABCA1 in mice increases plasma HDL cholesterol and reduces diet-induced atherosclerosis. Journal of lipid research. 2012; 53:158-67.

27. Lucero D, Sviridov D, Freeman L, Lopez GI, Fassio E, Remaley AT, Schreier L. Increased cholesterol efflux capacity in metabolic syndrome: relation with qualitative alterations in HDL and LCAT. Atherosclerosis. 2015; 242:236-42.

28. Ramirez CM, Davalos A, Goedeke L, Salerno AG, Warrier N, Cirera-Salinas D, Suarez Y, Fernandez-Hernando C. MicroRNA-758 regulates cholesterol efflux through posttranscriptional repression of ATP-binding cassette transporter A1. Arterioscler Thromb Vasc Biol. 2011; 31:2707-14.

29. O’Driscoll L, Kenny E, Mehta JP, Doolan P, Joyce H, Gammell P, Hill A, O'Daly B, O'Gorman D, Clynes M. Feasibility and relevance of global expression profiling of gene transcripts in serum from breast cancer patients using whole genome microarrays and quantitative RT-PCR. Cancer genomics \& proteomics. 2008; 5:94-104.

30. Knowles BB, Howe CC, Aden DP. Human hepatocellular carcinoma cell lines secrete the major plasma proteins and hepatitis B surface antigen. Science (New York, NY). 1980; 209:497-9.

31. Hennessy E, Clynes M, Jeppesen PB, O’Driscoll L. Identification of microRNAs with a role in glucose stimulated insulin secretion by expression profiling of MIN6 cells. Biochem Biophys Res Commun. 2010; 396:457-62.

32. Rottiers V, Naar AM. MicroRNAs in metabolism and metabolic disorders. Nature reviews Molecular cell biology. 2012; 13:239-50. 
33. He H, Chen $\mathrm{K}$, Wang $\mathrm{F}$, Zhao L, Wan X, Wang L, Mo Z. miR-204-5p promotes the adipogenic differentiation of human adipose-derived mesenchymal stem cells by modulating DVL3 expression and suppressing Wnt/ beta-catenin signaling. International journal of molecular medicine. 2015; 35:1587-95.

34. Davalos A, Goedeke L, Smibert P, Ramirez CM, Warrier NP, Andreo U, Cirera-Salinas D, Rayner K, Suresh U,
Pastor-Pareja JC, Esplugues E, Fisher EA, Penalva LO, et al. miR-33a/b contribute to the regulation of fatty acid metabolism and insulin signaling. Proceedings of the National Academy of Sciences of the United States of America. 2011; 108:9232-7.

35. Rotllan N, Fernandez-Hernando C. MicroRNA regulation of cholesterol metabolism. Cholesterol. 2012; 2012:847849. 